\title{
Non-iterative numerical implementation for the constitutive modelling of pressure-dependent elastoplasticity using paraboloidal yield criteria
}

\author{
V. Laheri ${ }^{1, *}$, F. A. Gilabert ${ }^{1}$ \\ ${ }^{1}$ Ghent University, Department of Materials, Textiles and Chemical Engineering \\ Technologiepark-Zwijnaarde 46, 9052 Zwijnaarde, Belgium. \\ * Corresponding author: vikram.laheri@ugent.be
}

\begin{abstract}
The approach to improve the mechanical performance of fibre-reinforced polymer composites at lower scales relies on micromechanical modelling of the representative volume elements combined with homogenisation techniques so that a detailed expression of the mechanical response can be provided. Besides the fibres, the mechanical response of the polymer matrix plays a crucial role in the overall composite performance. Due to the highly non-linear nature of polymers, a robust and consistent numerical treatment of its material model is a prerequisite.

The pressure-dependent plastic behaviour is an inherent feature in polymer matrices. The paraboloidal yield criterion postulated by [1] is adopted to describe pressure-dependent plasticity, which represents a paraboloid surface in the triaxial principal stress coordinates system. It meets the requirement for a closed surface avoiding angular apex in pure tensile and an open surface in pure compressive octants. The elastoplastic computational implementation developed by [2] computes plastic strain increment using a computationally demanding iterative approximation technique based on Newton-Raphson's method that does not always succeed under very large deformations.

This research aims at developing an efficient and exact implementation of the elastoplastic constitutive model with the paraboloidal plasticity theory. With this novel implementation, the evolution of plastic strain is computed by using a non-iterative scheme with unprecedented accuracy. This is achieved by solving the quadratic equation of the yield criterion described as a function of plastic strain increment, obtained using the update of trial stress from radial return mapping algorithm. This procedure diminishes the computational time and avoids the risk of aggregating approximation errors that lead to convergence solver issues. For verification, the proposed implementation is numerically investigated in three levels of benchmark examples: (i) simple single-element test to observe its functionality, (ii) cylinder compression test to analyse convergence issues and (iii) applicability of the implementation for realistic complex dog-bone geometry.
\end{abstract}

\section{REFERENCES}

[1] N. W. Tschoegl, 'Failure Surfaces in Principal Stress Space', Polymer Science Symposium, Vol. 32, pp. 239-267, 1971.

[2] A. R. Melro, P. P. Camanho, F. M. Andrade Pires, and S. T. Pinho, 'Micromechanical analysis of polymer composites reinforced by unidirectional fibres: Part I - Constitutive modelling', International Journal of Solids and Structures, Vol. 50, No. 11-12, pp. 18971905, 2013. 